\title{
Adaptive Shape based Interactive Approach to Segmentation for Nodule in Lung CT Scans
}

\author{
A. Sathesh, \\ Department of EEE, \\ Eritrea Institute of Technology, \\ Eritrea. \\ Email id: sathesh4you@gmail.com
}

\begin{abstract}
In lung cancer diagnosis, growth of pulmonary nodule should be detected perfectly. Mostly watershed segmentation methods play a very important role in lung CT images to detect their growth. But this method detection will be ineffective in terms of energy function and speed as well. The proposed modified graph-cut technique is providing the good performing result in the speed and accuracy of the process than the conservative graph cut methods. Also, this research paper is proposed adaptive shape based interactive approach to segmentation for lung CT scan image and provide a more efficient. This proposed algorithm is proving that the energy function of the system is lesser than old methods. In this research paper, applying shape-based technique in segmentation technique has been proposed and proved for better accuracy with low energy function.
\end{abstract}

Keywords: lung cancer, computed tomography, segmentation, adaptive shape prior.

\section{INTRODUCTION}

Lung cancer is the most deadly disease in world. The nodule in the lungs due to smoking, age, hemoptysis, and previous malignancy is very difficult to segment from the lungs. Since the medical images are having weak edges, most of the segmentation fails in this case.

Smoking and lung cancer (primary carcinoma of the lung) are both well documented but one of the most critical and less often touted facts the disease is that smoking is the big reason. Tobacco smoke also causes chronic lung disease and contributes to bladder, pancreas, and kidney cancers and it studies suggest that individuals who continue to smoke have medium to high pain, than those who are able to put cigarettes aside [1]. The primary objective of this research article is to detect and segment the nodule shape accurately. In addition, the segmentation is conscious about the less energy function. Shape based modified graph cut with adaptive technique is introduced here. Adaptive shape based interactive technique is combined with segmentation methods to get better results. The following radiographic parameters are used to justify the accuracy level of proposed technique;

- Size of the nodule

- Location of the nodule

- Edge

- Growth rate

\section{RELATED WORK}

This paper presents a shape based curve growing algorithm for object recognition in medical image. Previously the curve growing process is constructed with the help of Bayesian network which will influence by fixed data base of the shape of the curve [2]. The solution of maxmum a posteriori is derived through energy diminish tool. It is executed in an adaptive regularization construction that stabilizes the influence of image data and shape based in estimating the curve, and reflects the fundamental dependencies in the Bayesian network. This method assuages over-

ISSN: 2582-2640 (online)

Submitted: 06.12.2020

Accepted: 20.12.2020

Published: 28.12.2020 
Journal of Soft Computing Paradigm (JSCP) (2020)

Vol.02/ No.04

Pages: 216-225

http://irojournals.com/jscp/

DOI: https://doi.org/10.36548/jscp.2020.4.003

smoothing in an effective manner, which can take place with other regularization methods [3]. This proposed construction addresses initialization and local min-max factors. Robustness and performance of the proposed method are experimented by segmentation of pulmonary fissures in computed tomography (CT) images [4]. Even star shape prior segmentation method also fails to detect weak edges in noisy environment. [12, 13, and 14].

\section{PROPOSED METHOD}

The pre-processing algorithm has written and it is followed by the proposed algorithm for the segmentation.

\section{STEP 1}

1(a) Input image $P_{1}$ (read the original input image)

1(b) Opening: $P_{1}=>$ eroded image $P_{2}=>$ dilated image $P_{3}$ (erode and then dilate the original image to get the Opening image)

1(c) Closing: $\mathrm{P1}=>$ dilated image $\mathrm{P}_{4}=>$ eroded image $\mathrm{P}_{5}$ (dilate and then erode the original image to get the Opening image)

1(d) Top hat: $\mathbf{P 6}<=\mathrm{P}_{1}-\mathrm{P}_{3}$ (subtract the opening image from the original image)

1(e) Bottom hat: $P 7<=P_{5}-P_{1}$ (subtract the original image from the closing image)

1(f) $\quad \mathbf{P}_{1}+\mathbf{P}_{6} \Leftrightarrow \mathbf{P}_{8}-\mathbf{P}_{7} \Rightarrow \mathbf{P}_{9}$ (superimpose the original and top hat image and subtract the Bottom hat image from the output image)

1(g) $\quad P_{9}=>$ enhanced image (get the output as enhanced image for pre-processing)

\section{STEP 2}

Initially, the graph cut was constructed for the segmentation approach in the natural images which was employed already [5]. Usually, the graph cut methods are generating energy function from the region and boundary information of the image.

2(a) Calculate the Energy Function E(A).

2(b) Change the value of the Regularizing parameter $\omega$ by iterations.

2(c) Find out the region and boundary parameter values.

This energy function can be represented as,

Where,

$$
\mathrm{E}(\mathrm{A})=\omega \mathrm{R}(\mathrm{A})+\mathrm{B}(\mathrm{A})
$$

$$
R(A)=\omega \sum_{P \in I} R\left(P, A_{P}\right)(\text { region })
$$

I will consider as set of pixel in an image with neighbours denoted as $\mathrm{N}$ given as [p,q]. The label assigned to pixel $p \& q$ as $A_{p} \& A_{q}$ respectively.

$$
B(A)=\sum_{\{p, q\} \in N} B(p, q) \cdot \delta A_{p} \neq A_{q}(\text { boundary terms })
$$

and

$$
\delta_{A p \neq A q}=\left\{\begin{array}{l}
1 \text { if } A_{p} \neq A_{q} \\
0 \text { if } A_{p}=A_{q}
\end{array}\right\}
$$

The co-efficient $\omega$ is the normalizing constraint $\omega \geq 0$. The region defined $R\left(P, A_{p}\right)$ as follows,

$$
\mathrm{R}\left(\mathrm{P}, \mathrm{A}_{\mathrm{P}}\right)=-\ln \operatorname{pr}\left(\mathrm{IA}_{\mathrm{P}} / \mathrm{A}_{\mathrm{P}}\right)
$$

ISSN: 2582-2640 (online)

Submitted: 06.12.2020

Accepted: 20.12.2020

Published: 28.12.2020 
Journal of Soft Computing Paradigm (JSCP) (2020)

Vol.02/ No.04

Pages: 216-225

http://irojournals.com/jscp/

DOI: https://doi.org/10.36548/jscp.2020.4.003

Where $I_{A P}$ is strength of the pixel $p$, and $\operatorname{prob}\left(I_{P} / A_{P}\right)$ is the probability of the intensity $I A_{P}$ given the Label $A_{P}$. The information of boundary is given by,

$$
\mathrm{B}(\mathrm{p}, \mathrm{q})=\exp \left(-\left(\mathrm{IAP}_{\mathrm{P}-\mathrm{IAq}}\right)^{2} *(|\mathrm{p}-\mathrm{q}|)\right) / 2 \sigma^{2}
$$

In an equation $6, \sigma$ note the noise standard deviation while $\|p-q\|$ as Euclidean distance between pixel $p$ and pixel $\mathrm{q}$ in the image.

\section{Remarks:}

In those equations, the nodes are considered for energy function in three different nodes such as pixel, object and background [6]. We have assumed here pixel node is connected with object node and boundary node connected with edge weighting factor. So the min cut and max cut flow can drop or minimize the energy function. The parameter $\omega$ is not easy to regularize or modify of it in the graph cut methods.

\section{STEP 3}

In order to solve the problem associated with the graph-cut method in terms of $\omega$, new techniques are introduced this new approach completes precise in the segmentation absence of associate the regularizing parameter [7]. This new approach contains boundary factor alone. Due to holding the region information in the conservative graph cut method is having more in energy function. Our new approach reduces the energy function during the process. This form shows in equation 7.

3(a) Remove the region parameter from the energy function.

3(b) Calculate the energy function using boundary parameter.

$$
E_{P(L)}=\sum_{\{p, q\} \in 1 N} B(p, q) \delta\left(L_{p} \neq L_{q}\right) F_{C}(p, q)
$$

Where $\mathrm{F}_{\mathrm{c}}(p, q)$ specifies consequences of the pixel $\mathrm{p}$ and $\mathrm{q}$ are contain to the same label.

$$
\mathrm{F}_{\mathrm{c}}(p, q)=\operatorname{prob}\left(\mathrm{IA}_{\mathrm{P}} / \mathrm{A}_{\mathrm{P}=0}\right) \operatorname{prob}\left(\mathrm{IA}_{\mathrm{q}} / \mathrm{A}_{\mathrm{q}=0}\right)+\operatorname{prob}\left(\mathrm{IA}_{\mathrm{P}} / \mathrm{A}_{\mathrm{P}=-1}\right) \operatorname{prob}\left(\mathrm{IAs}_{\mathrm{q}} / \mathrm{A}_{\mathrm{q}=1}\right)
$$

The object background intensity histograms should have the pixel p probability with that. It shows in the equation wise,

$$
\operatorname{prob}\left(\mathrm{IA}_{\mathrm{P}} / \mathrm{A}_{\mathrm{P}=1}\right) \text { and }\left(\mathrm{IA}_{\mathrm{q}} / \mathrm{A}_{\mathrm{q}=0}\right)
$$

\section{Remarks:}

As we expected, the size of the memory required is reduced even though there is no regional information. This approach can apply the mixture module for colour images. When handling the colour images, the following model should be appended with the algorithm.

$$
I A_{P}=\Pi^{T} A_{t}\left(C_{P}\right)
$$

Where " $\Pi$ " is indicating the transformation factor to grey scale.

\section{STEP 4}

The reduction of problem occurs in the image as a noise and smear in the conservative methods;

4(a) Combine the energy function of graph cut and shape prior method. 4(b) Find out the number of iterations to meet the shape.

ISSN: 2582-2640 (online)

Submitted: 06.12.2020

Accepted: 20.12.2020

Published: 28.12.2020 
Journal of Soft Computing Paradigm (JSCP) (2020)

Vol.02/ No.04

Pages: 216-225

http://irojournals.com/jscp/

DOI: https://doi.org/10.36548/jscp.2020.4.003

\section{Remarks:}

In order to get the original energy function for this process is given by,

$$
E=E_{i}+\alpha E_{s}
$$

Where $E_{\mathrm{i}}$ is exist energy in the image, $\mathrm{E}_{\mathrm{s}}$ is the shape prior energy and $\alpha$ is a constant. It is used to maintain the balance between exit energy and shape prior energy. The shape prior method is improving in the energy function in the process. The result of adaptively shape prior has written in the equation 12 below.

$$
\begin{aligned}
& E=\sum_{p \in i} D_{p}\left(f_{p}\right)+\sum(p, q) \in N: F_{p} \neq F_{q} v_{p q}\left(F_{p}, F_{q}\right)+ \\
& \sum(p, q) \in N: f_{q} \frac{1}{e(\alpha P-\alpha q)^{2}} S_{p q}\left(F_{p}, F_{q}\right)
\end{aligned}
$$

\section{STEP5}

During the implementation process of proposing methods consumes lot of memory space compared with exist methods of segmentation.

\section{5(a) Combine the energy function of improved graph cut and shape based method.}

5(b) Find out the number of iterations to meet the shape.

\section{Remarks:}

In order to reduces the usage of more energy function by improved version of graph cut algorithm, the new energy function can be modified and written as,

$$
E(G)=E_{m}+E_{A}
$$

$E_{m}$ is changed energy function for graph cut segmentation which is very small. Where $E_{A}$ is the energy function of adaptively shape based method.

$$
E_{m}=\sum_{\{p, q\} \in N} B(p, q) \delta\left(L_{p} \neq L_{q}\right) F_{c}(p, q)
$$

The parameters in the equation are as explained in the previous section.

$$
E_{A}=\sum(p, q) \in N: f_{p} \neq f_{q} \frac{1}{e(\alpha p-\alpha q)^{2}} E_{p q}\left(f_{p}, f_{q}\right)
$$

Where $\alpha p$ represents the probability value from the map in pixel $p . E_{p q}\left(f_{p}, f_{q}\right)$ represents a pair wise shape based preset at the pixels $\mathrm{p}$ and $\mathrm{q}$. The kernel method algorithm also gives more similarity with adaptively shape based method such as functional analysis and process of filtering and so on. Finally our proposed adaptively shape based method is derived and written below,

ISSN: 2582-2640 (online)

Submitted: 06.12.2020

Accepted: 20.12.2020

Published: 28.12.2020 
Journal of Soft Computing Paradigm (JSCP) (2020)

Vol.02/ No.04

Pages: 216-225

http://irojournals.com/jscp/

DOI: https://doi.org/10.36548/jscp.2020.4.003

$$
\begin{aligned}
& E(G)=\sum_{\{P, Q\} \in N} B(p, q) \delta\left(L_{P} \neq L_{q}\right) F_{C}(p, q)+ \\
& \sum(p, q) \in N: F_{P} \neq F_{q} * \frac{1}{e(\alpha p-\alpha q)^{2}} E_{p q}\left(F_{p}, F_{q}\right)
\end{aligned}
$$

The probability of malignancy has been calculated based on the following parameters. It includes the clinical properties and radiographic properties.

\section{PROBLEM STATEMENT WITH BAYES THEOREM}

For growth rate prediction, the Bayes' theorem describes the relationships that exist within an array of simple and conditional probabilities [8]. This theorem can help here that the probability is taking charge of new piece of evidence. It has been widely used many areas such as in marine biology and spam blockers in e-mails [9]. Bayes theorem is used to find falsification and relation between the two probabilities and more precise, and sometimes extended or corrected. The performance analysis is performed by creating a problem statement. The values are calculated by using baye's theorem.

There are 100 patients having smoking behaviour, depending upon their size of the nodule, Doctor divided them into two categories as 5 and 95 respectively [10]. He predicted that there is a possibility of $90 \%$ cancer in the first 5 patients and $10 \%$ cancer in the next 95 patients.

According to Bayes theorem,

$$
\mathrm{P}(\mathrm{A} 1 \mid \mathrm{B})=\frac{\mathrm{P}(\mathrm{A} 1) \mathrm{P}(\mathrm{B} \mid \mathrm{A} 1)}{\mathrm{P}(\mathrm{A} 1) \mathrm{P}(\mathrm{B} \mid \mathrm{A} 1)+\mathrm{P}(\mathrm{A} 2) \mathrm{P}(\mathrm{B} \mid \mathrm{A} 2)}
$$

Consider,

Event A1 (Patients having more symptoms)

Event A2 (Patients having less symptoms)

Event B (Prediction of cancer by Doctor)

Table 1: Probability prediction

\begin{tabular}{|c|c|c|c|c|c|c|c|}
\hline $\mathrm{P}(\mathrm{A} 1)$ & \multicolumn{7}{|c|}{$5 / 100=0.05$} \\
\hline $\mathrm{P}(\mathrm{A} 2)$ & \multicolumn{1}{|c|}{$95 / 100=0.95$} \\
\hline $\mathrm{P}(\mathrm{B} / \mathrm{A} 1)$ & 0.2 & 0.3 & 0.4 & 0.5 & 0.6 & 0.7 & 0.8 \\
\hline $\mathrm{P}(\mathrm{B} / \mathrm{A} 2)$ & 0.8 & 0.7 & 0.6 & 0.5 & 0.4 & 0.3 & 0.2 \\
\hline
\end{tabular}

Table 1 shows the probability of the events A1, A2 respectively. The probabilities predicted by medical practitioners based on the symptoms are $\mathrm{P}(\mathrm{B} \mid \mathrm{A} 1)$ and $\mathrm{P}(\mathrm{B} \mid \mathrm{A} 2)$. Using the formulas,

$\mathrm{P}(\mathrm{A} 1 \mid \mathrm{B})=\frac{\mathrm{P}(\mathrm{A} 1) \mathrm{P}(\mathrm{B} \mid \mathrm{A} 1)}{\mathrm{P}(\mathrm{A} 1) \mathrm{P}(\mathrm{B} \mid \mathrm{A} 1)+\mathrm{P}(\mathrm{A} 2) \mathrm{P}(\mathrm{B} \mid \mathrm{A} 2)}$ And $\mathrm{P}(\mathrm{A} 2 \mid \mathrm{B})=\frac{\mathrm{P}(\mathrm{A} 2) \mathrm{P}(\mathrm{B} \mid \mathrm{A} 2)}{\mathrm{P}(\mathrm{A} 1) \mathrm{P}(\mathrm{B} \mid \mathrm{A} 1)+\mathrm{P}(\mathrm{A} 2) \mathrm{P}(\mathrm{B} \mid \mathrm{A} 2)}$

The conditional probabilities by bayes theorem have been calculated and show in the Table 1 .

ISSN: 2582-2640 (online)

Submitted: 06.12.2020

Accepted: 20.12.2020

Published: 28.12.2020 
Journal of Soft Computing Paradigm (JSCP) (2020)

Vol.02/ No.04

Pages: 216-225

http://irojournals.com/jscp/

DOI: https://doi.org/10.36548/jscp.2020.4.003

Table 2: Comparison of probability prediction

\begin{tabular}{|c|c|c|c|}
\hline $\begin{array}{c}\text { Medical practitioner's } \\
\text { prediction } \\
(\text { In \%) }\end{array}$ & \multicolumn{2}{c|}{$\begin{array}{c}\text { Bayes theorem probability } \\
\text { prediction } \\
\text { (In \%) }\end{array}$} \\
\hline 5 patients & 95 patients & 5 patients & 95 patients \\
\hline $\mathbf{3 0}$ & $\mathbf{7 0}$ & $\mathbf{2}$ & $\mathbf{9 7}$ \\
\hline $\mathbf{4 0}$ & $\mathbf{6 0}$ & $\mathbf{3}$ & $\mathbf{9 6}$ \\
\hline $\mathbf{5 0}$ & $\mathbf{5 0}$ & $\mathbf{5}$ & $\mathbf{9 5}$ \\
\hline $\mathbf{6 0}$ & $\mathbf{4 0}$ & $\mathbf{7}$ & $\mathbf{9 2}$ \\
\hline $\mathbf{7 0}$ & $\mathbf{3 0}$ & $\mathbf{1 0}$ & $\mathbf{8 9}$ \\
\hline $\mathbf{8 0}$ & $\mathbf{2 0}$ & $\mathbf{1 7}$ & $\mathbf{8 2}$ \\
\hline
\end{tabular}

Table 2 clearly shows that the prediction of medical practitioner is almost wrong in all the cases, if the prediction is correct, then it would be same as $50 \%$ for 5 patients and $50 \%$ for 95 patients, but the results obtained are $5 \%$ and $95 \%$ for 5 patients and 95 patients respectively.

From these results, we can conclude that the size plays important role more than that of the symptoms and the medical practitioner's prediction. In order to predict the cancer in the very early stage, segmentation of the nodule is essential [11].

\section{RESULTS AND DISCUSSIONS}

Adaptively shape based interactive approach is comparing with improved version of segmentation methods. Our proposed method is obtained positive response in energy function and accuracy level rises. The obtained results show in figures below. Mostly, the proposed method is similar to or better than the pre-existing methods and the speed of operation in user initialization.

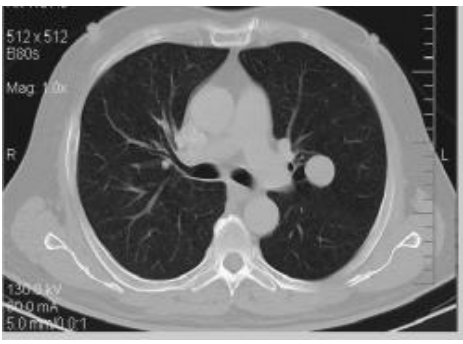

(a)

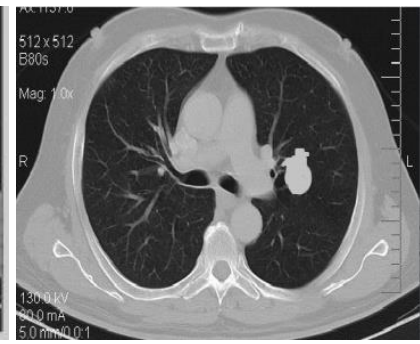

(b)

Fig 1 (a), (b) Original images

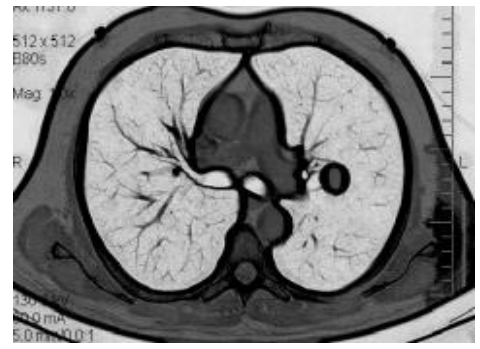

(a)

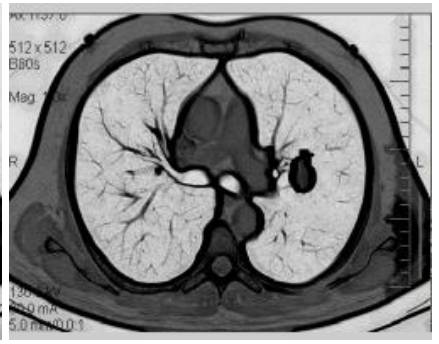

(b)

Fig 2 (a), (b) Enhanced images

ISSN: 2582-2640 (online)

Submitted: 06.12.2020

Accepted: 20.12.2020

Published: 28.12.2020 
Journal of Soft Computing Paradigm (JSCP) (2020)

Vol.02/ No.04

Pages: 216-225

http://irojournals.com/jscp/

DOI: https://doi.org/10.36548/jscp.2020.4.003

The various nodule sized input CT scan images are shown in Fig 1, a \& b and the preprocessing has been done for all the images and the enhanced images are shown in Fig 2, a \& b the following figures show the output of different segmentation methods.

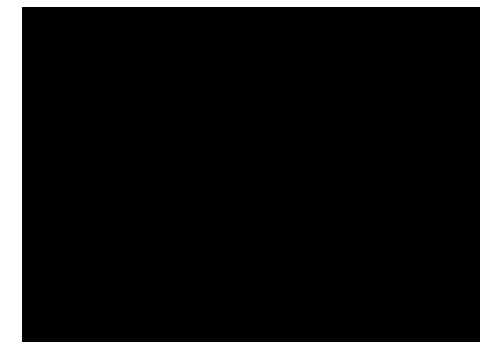

(a)

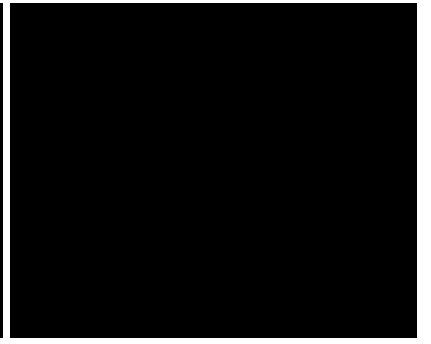

(b)

Fig 3 (a), (b) Graph cut segmented images

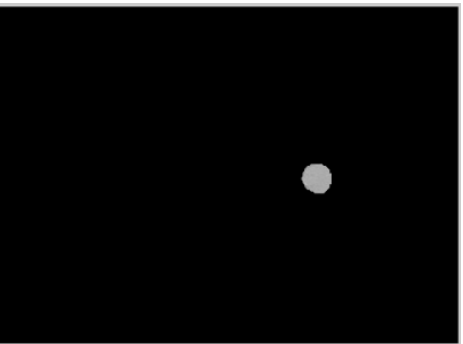

(a)

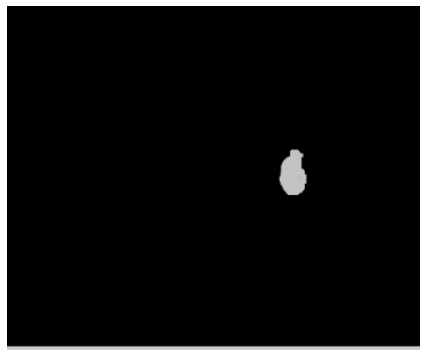

(b)

Fig 4 (a), (b) Improved version of graph cut segmented images

The above figure 4 shows the results of improved version of graph cut segmented from the given image. Our proposed adaptively shape based interactive approach for segmentation process contain the less energy function and better accuracy shows through the visual representation which is agreed by many medical practitioners. The final result shown in the figure 5 (a) \& (b) is the output of our proposed technique.

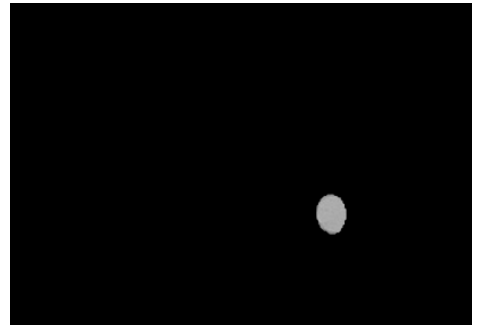

(a)

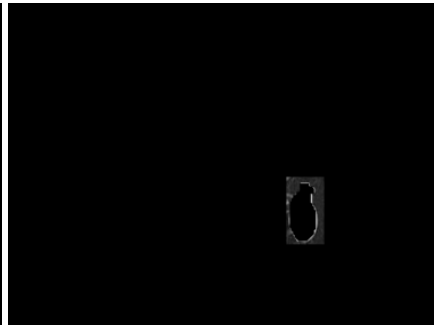

(b)

Fig 5 (a), (b) adaptive shape based graphcut segmented images

ISSN: 2582-2640 (online)

Submitted: 06.12.2020

Accepted: 20.12.2020

Published: 28.12.2020 
Journal of Soft Computing Paradigm (JSCP) (2020)

Vol.02/ No.04

Pages: 216-225

http://irojournals.com/jscp/

DOI: https://doi.org/10.36548/jscp.2020.4.003

Table 3 Comparison of energy function of segmented outputs

\begin{tabular}{|c|c|c|}
\hline $\begin{array}{c}\text { Graph Cut } \\
\text { Segmentation }\end{array}$ & $\begin{array}{c}\text { Modified GraphCut } \\
\text { Segmentation }\end{array}$ & $\begin{array}{c}\text { Proposed Adaptively } \\
\text { Shape based } \\
\text { Segmentation }\end{array}$ \\
\hline 3889.334723 & 3810.909675 & 3731.23475 \\
3693.144915 & 3614.980532 & 3535.233532 \\
3592.681492 & 3460.258979 & 3460.258979 \\
3415.190446 & 3346.396535 & 3106.875634 \\
3233.460124 & 3231.467134 & 3088.34566 \\
3046.590653 & 3105.716309 & 3055.342128 \\
2938.147069 & 2921.644583 & 2901.766894 \\
2798.168804 & 2796.216365 & 2607.45676 \\
2672.181764 & 2614.787883 & 2548.794746 \\
2492.174916 & 2490.435386 & 2395.444747 \\
2349.033533 & 2297.509024 & 2157.568478 \\
2162.510647 & 2157.968773 & 2023.34940 \\
\hline
\end{tabular}

The above table 3 shows the energy function between graph cut methods. The comparisons of the improved version of segmentation method and adaptive shape prior segmentation are shown in the below pictures in different locality of nodule. The output results are shown to prove adaptive shape prior works well in this case. Improved version of graph cut algorithm fails to detect the weak edges of the CT image.

It is clear from the results that the adaptive shape prior segments the nodule exactly. It is capable of segmenting and detecting the weak edges which modified graph cut fails to segment it properly. Though the results of conservative segmentation are not satisfied, the improved version of graph cut segmentation technique with adaptive shape prior is been implemented.

The figure 5 and 6 shows the different location of nodule and experimented with our proposed algorithm. It shows the superior than others in terms of energy function, accuracy, less memory storage containing.

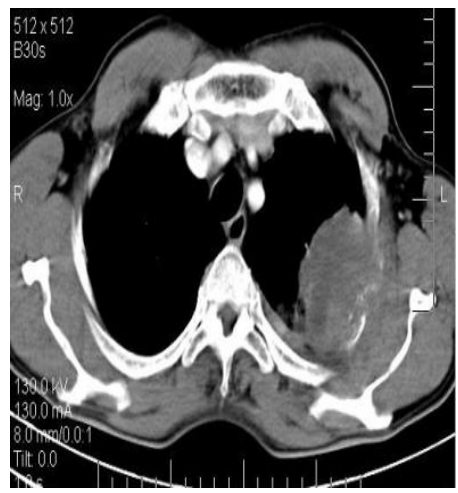

(a)

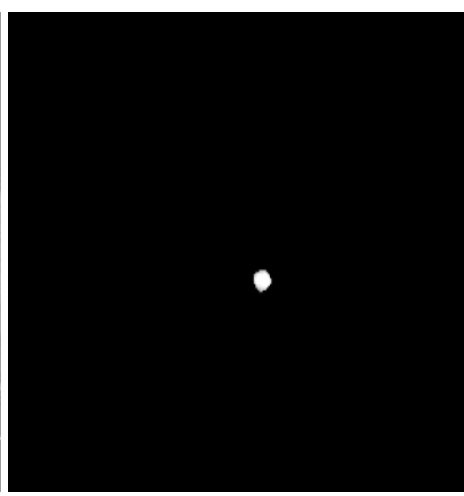

(b)

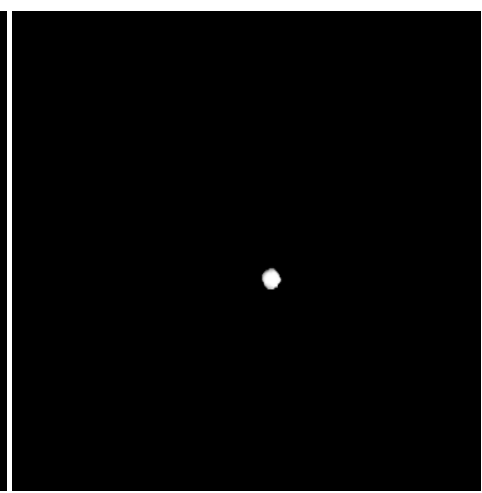

(c)

Fig 5 (a) Original image1, (b) Modified graph cut segmented image1, (c) Adaptive shape prior segmented image1

ISSN: 2582-2640 (online)

Submitted: 06.12.2020

Accepted: 20.12.2020

Published: 28.12.2020 


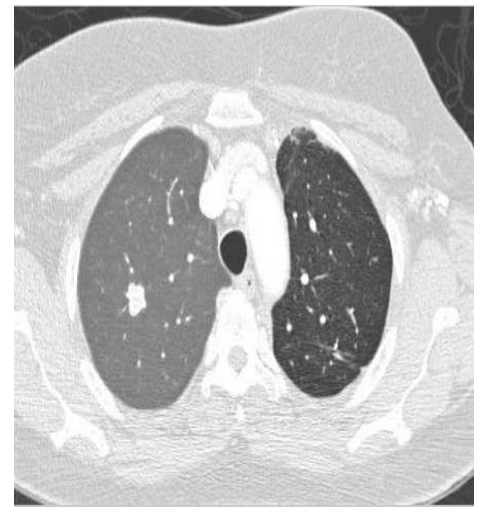

(a)

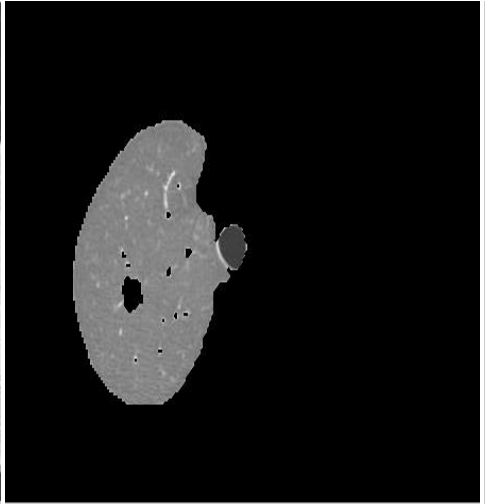

(b)

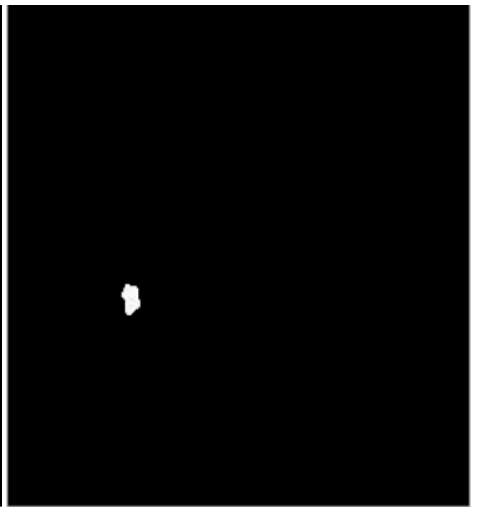

(c)

Fig 6 (a) Original image2,(b) Modified graph cut segmented image2, (c) Adaptive shape prior segmented image2

This proposed method is used to reduce the size of the memory required and lesser energy function in this process as well as. Moreover, this proposed method uses to minimize the problems arises such as not identifying weak edges, abrupt noise interruption in improved version of graph cut segmentation method.

\section{CONCLUSION}

Though conservative methods are not satisfied; in order to handle the low energy function and occupy the less memory space in the system, proposed here adaptive shape based interactive approach to segmentation methods. This proposed method is proved very accurate and efficient than improved version of graph cut methods in segmentation techniques.

From the obtained results, adaptive shape prior with adaptively changed graph cut is concluded as best. From table 3 , it is clear that the energy function for this method is lesser than that of improved version of graph cut segmentation and conservative graph cut segmentation. If noise is affected more in this picture its energy function, accuracy level is dropping down. Further development of this algorithm is that the improving of accuracy and efficient level in giant noisy environment.

\section{ACKNOWLEDGEMENT}

We would like to thank the Consultant Diagnostic Radiologist \& Sonologist Dr. V. Prabakarakumar MBBS, DMRD of Best Scan Centre Coimbatore for his great support to complete this paper. We would like to extend our thank Ms.Shanmuga Priya who did survey for this research paper.

\section{REFERENCES}

[1] Ahmed Soliman et al"Accurate Lungs Segmentation on CT Chest Images by Adaptive Appearance-Guided Shape Modeling" Published in IEEE Transactions on Medical Imaging PP(99):1-1 September 2016 DOI: 10.1109/TMI.2016.2606370.

[2] Furqan Shaukat et al "An Efficient Algorithmic Solution for automatic Segmentation of Lungs from CT images" Published in Pakistan journal of science 70(1):71-8 March 2018

[3] D. L. Pham, C. Y. Xu, and J. L Prince, "Current Methods in Medical Image Segmentation," in Annual Review of Biomedical Engineering, Vol. 2, Pages: 1-5, 2000.

[4] I. Sluimer, P. Waes, M. Viergever, and B. Ginneken, "Computer-aided diagnosis in high resolution CT of the lungs," Med. Phys. 30, 3081-3090. 2003.

[5] J. Keller, F. Edwards, and R. Rundle, "Automatic outlining of regions on CT scans," J. Comput. Assist. Tomogr. 5, 240-245.1981.

ISSN: 2582-2640 (online)

Submitted: 06.12.2020

Accepted: 20.12.2020

Published: 28.12.2020 
Journal of Soft Computing Paradigm (JSCP) (2020)

Vol.02/ No.04

Pages: 216-225

http://irojournals.com/jscp/

DOI: https://doi.org/10.36548/jscp.2020.4.003

[6] R. Haralick, "Statistical and structural approaches to texture," Proc. IEEE 67, 786-804 1979.International Journal of Computer and Electrical Engineering, Vol. 3, No. 6, December 2011.

[7] Yamomoto, S., Jiang, H., Matsumoto, M., Tateno, Y., Iinuma, T., Matsumoto, "Image Processing for Computer- Aided Diagnosis of Lung Cancer by CT (LSCT)", IEEE Workshop on Applications of Computer Vision, pp. 236 - 241, 1996.

[8] Yeny Yim, Helen Hong, Yeong Gil Shin, "Hybrid Lung Segmentation in Chest CT Images for ComputerAided Diagnosis", International Workshop on Enterprise networking and Computing in Healthcare Industry, Pp. $378-383,2005$.

[9] Zhi-Hua Zhou, et al.,"Lung Cancer Cell Identification Based on Artificial Neural Network Ensembles", Elsevier Science 24(1): p. 25-36 (2002).

[10] Y. Boykov and G. Funka-Lea. Graph cuts and efficient n-d image segmentation. Int'1 J. Computer Vision, 70(2):109-131, Nov. 2006.

[11] D. Freedman and T. Zhang, "Interactive graph cut based segmentation with shape priors," in IEEE Conference on Computer Vision and Pattern Recognition, 2005, pp. 755-762.

[12] O. Veskler, "Star shape prior for graph-cut image segmentation," in IEEE European conference on Computer Vision, 2008, pp. 454-467.

[13] G. Slabaugh and G. Unal, "Graph cuts segmentation using an elliptical shape prior," in International Conference on Image Processing, 2005, vol. 2.

[14] N. Vu and B. S. Manjunath, "Shape prior segmentation of multiple objects with graph cuts," in Proceedings of the IEEE Conference on Computer Vision and

Pattern Recognition, 2008, pp. 1-8.

ISSN: 2582-2640 (online)

Submitted: 06.12.2020

Accepted: 20.12.2020

Published: 28.12.2020 